\title{
Upregulated miRNA-182-5p expression in tumor tissue and peripheral blood samples from patients with non-small cell lung cancer is associated with downregulated Caspase 2 expression
}

\author{
$\mathrm{LU} \mathrm{YANG}^{1 *}, \mathrm{YE} \mathrm{DOU}^{2 *}$, ZHUXIN SUI ${ }^{2}, \mathrm{HUI} \mathrm{CHENG}^{2}, \mathrm{XIA} \mathrm{LIU}^{2}$, \\ QINGLU WANG ${ }^{2}$, PEIFU GAO ${ }^{2}$, YIN'E $^{2} U^{3}$ and MING XU ${ }^{2}$

\begin{abstract}
Departments of ${ }^{1}$ Pathology and Pathophysiology and ${ }^{2}$ Human Anatomy, Histology and Embryology,
Teaching Department of Basic Medicine, Qilu Medical University, Zibo, Shandong 255213;

${ }^{3}$ Department of Histology and Embryology, School of Basic Medicine, North China University
\end{abstract} \\ of Science and Technology, Tangshan, Hebei 063210, P.R. China
}

Received July 3, 2018; Accepted January 10, 2019

DOI: $10.3892 /$ etm.2019.8074

\begin{abstract}
Lung cancer has the highest morbidity and mortality rates among all malignant tumors worldwide. Previous studies demonstrated that microRNA (miR)-182-5p may serve different roles in different types of cancer, including renal cell carcinoma and liver cancer. However, the functional role of miR-182-5p in non-small cell lung cancer (NSCLC) remains unknown. In the current study, the expression level of miR-182-5p in tumor tissue and peripheral blood samples obtained from patients with NSCLC was examined. The biological function of miR-182-5p on NSCLC cell proliferation was also investigated. Tissue and adjacent normal tissue samples were collected from 33 patients with NSCLC. In addition, peripheral blood samples were obtained from patients with NSCLC and 26 healthy control patients. The NSCLC cell line H1299 was used for all functional assays. Reverse transcription-quantitative polymerase chain reaction was used to determine the miR-182-5p or Caspase 2 (CASP2) mRNA expression levels in NSCLC tissue and peripheral blood samples, as well as in the NSCLC cell line. Western blotting was used to examine the protein expression level of CASP2 in tissue samples and cells, and ELISA was performed to measure the protein level of CASP2 in peripheral blood samples. MTT assay was performed to examine NSCLC cell proliferation. Flow cytometry was used to detect apoptosis. Dual-luciferase reporter assay was used to
\end{abstract}

Correspondence to: Dr Ming Xu, Department of Human Anatomy, Histology and Embryology, Teaching Department of Basic Medicine, Qilu Medical University, 246 West Guojing Road, Zibo, Shandong 255213, P.R. China

E-mail: qlyyxyxm@163.com

*Contributed equally

Key words: microRNA-182-5p, caspase 2, non-small cell lung cancer, proliferation examine whether miRN182-5p directly interacts with CASP2. The current study demonstrated that miR-182-5p expression was upregulated in NSCLC tissue and peripheral blood samples from patients with NSCLC, which suggests that miR-182-5p, may serve a functional role in NSCLC. In addition, inhibition of miR-182-5p expression suppressed cell proliferation and enhanced cell apoptosis in NSCLC cells. CASP2 expression was downregulated in NSCLC tissue and peripheral blood samples from patients with NSCLC. The current study demonstrated that miR-182-5p may regulate NSCLC cell proliferation and apoptosis by regulating CASP 2 expression as miR-182-5p directly binds with the 3'-untranslated region of CASP2, thereby regulating CASP2 expression.

\section{Introduction}

Lung cancer has the highest morbidity and mortality rates among all malignant tumors worldwide (1). Lung cancer accounts for $\sim 13 \%$ of all new cancer cases each year $(2,3)$. In China, $\sim 600,000$ people succumb to lung cancer every year $(4,5)$, and therefore early prevention and diagnosis of lung cancer is important. The current treatment strategy for lung cancer is mainly surgery and chemoradiotherapy; targeted therapy is an emerging treatment strategy (6).

MicroRNAs (miRNA or miR) is a class of small, non-encoding RNA molecules (7). miRNAs serve important roles in several biological and pathological processes by promoting mRNA degradation or inhibiting mRNA translation, to negatively regulate target gene expression (8). Certain miRNA molecules can function as oncogenes or tumor-suppressors and, together with their target genes, can serve important roles in the occurrence and development of several types of cancer (7). Previous studies identified the important role of miRNAs in non-small cell lung cancer (NSCLC), as several aberrantly expressed miRNAs were identified in NSCLC tissue samples $(9,10)$. Previous studies demonstrated that miR-182-5p may serve different roles in different types of cancer, including renal cell carcinoma and liver cancer $(11,12)$. However, the functional role of miR-182-5p in NSCLC remains unknown. 
Caspase 2 (CASP2) may function in stress-induced cell death pathways, cell cycle maintenance and tumor suppression (13). CASP2 can induce apoptosis, and overexpression of CASP2 was previously demonstrated in several types of cells $(14,15)$. CASP2 is regulated by several miRNAs, such as miR-183 in ovarian cancer (16). In the case of a calorie-restricted diet, CASP2 in liver cells has been shown to be precisely regulated by miR-125a-5p, which delays aging (17). The aforementioned studies shown that the regulation of CASP2 by miRNAs has become one of the important factors affecting the expression of CASP2.

In the current study, bioinformatics analysis was used to identify CASP2 as a potential target gene of miR-182-5p, however the association between CASP2 and miR-182-5p has not yet been reported. The current study demonstrated that miR-182-5p expression is upregulated, while CASP2 expression is downregulated in NSCLC tissue and peripheral blood samples from patients with NSCLC. Therefore, miR-182-5p may regulate NSCLC cell proliferation and apoptosis via direct interaction with CASP2.

\section{Materials and methods}

Patient samples. NSCLC tissue and adjacent normal tissue samples were collected from 33 patients (male, $n=21$ and female, $n=12$; age range, 36-68 years; median age, 51 years) who had undergone surgical resection surgery at Department of Pathology and Pathophysiology, Zibo Zhoucun People's Hospital Affiliated to Qilu Medical University (Zibo, China) between December 2013 and December 2017. None of the patients received any prior treatment with hormones, Chinese medicines or chemoradiotherapy. In addition, 26 healthy control patients (male, $n=16$ and female, $n=10$; age range, 35-72 years; median age, 52 years) who had undergone physical examinations at Zibo Zhoucun People's Hospital Affiliated to Qilu Medical University between December 2013 and December 2017 were included in the study. Peripheral blood was collected from patients with NSCLC and healthy controls. The current study was approved by the Ethics Committee of Qilu Medical University and written informed consent was obtained from all patients or their families.

Cell culture and transfection. The NSCLC cell line H1299 was purchased from the Cell Bank of the Chinese Academy of Sciences (Shanghai, China), and cultured in high-glucose Dulbecco's modified Eagle's medium (DMEM; Hyclone; GE Healthcare Life Sciences, Little Chalfont, UK) supplemented with $10 \%$ fetal bovine serum (FBS; Hyclone; GE Healthcare Life Sciences), $100 \mathrm{IU} / \mathrm{ml}$ penicillin and $100 \mathrm{IU} / \mathrm{ml}$ streptomycin. Cells were maintained at $37^{\circ} \mathrm{C}$ in a $5 \% \mathrm{CO}_{2}$ incubator with $70 \%$ humidity. The cells were passaged every 4 days, and log-phase cells were collected for further experimentation.

H1299 cells were seeded into 24-well plates at a density of $3 \times 10^{5}$ cells/well and cultured in antibiotic-free DMEM supplemented with $10 \%$ FBS until $70 \%$ confluent. In a vial, $1 \mu 1 \mathrm{miR}-182-5 \mathrm{p}$ inhibitor $(20 \mathrm{pmol} / \mathrm{ml}$; 5'-AAACCGUUA CCAUCUUGAGUGUGA-3') with CASP2 small interfering (si)RNA (siCASP2; forward, 5'-ACAGCUGUUGUUGAG CGAAdTdT-3'; reverse, 5'-UUCGCUCAACAACAGCUG UdTdT-3') or siNC (forward, 5'-UUCUCCGAACGUGUC
ACGUdTdT-3'; reverse, 5'-ACGUGACACGUUCGGAGAAdT dT-3'; both 20 pmol $/ \mu 1$; Sangon Biotech Co., Ltd., Shanghai, China) were mixed with $50 \mu$ l Opti-MEM medium (Thermo Fisher Scientific, Inc., Waltham, MA, USA). In a second vial, $1 \mu 1$ Lipofectamine ${ }^{\circledR} 2000$ (Thermo Fisher Scientific, Inc.) was mixed with $50 \mu \mathrm{l}$ Opti-MEM medium. Following $5 \mathrm{~min}$ incubation at room temperature, the two vials were combined and incubated for a further $20 \mathrm{~min}$ at room temperature. The transfection mixture was subsequently added to cells and following $6 \mathrm{~h}$ incubation at $37^{\circ} \mathrm{C}$, the medium was replaced with F12/DMEM supplemented with 10\% FBS. Following 48-h transfection, cells were collected and used in further experimentation.

Reverse transcription-quantitative polymerase chain reaction (RT-qPCR). Tissue samples $(100 \mathrm{mg})$ were ground into powder in liquid nitrogen. Total RNA was extracted from powdered tissue samples, plasma $(100 \mu \mathrm{l})$ or cells $\left(3 \times 10^{6}\right)$ using $1 \mathrm{ml}$ TRIzol ${ }^{\circledR}$ reagent (Thermo Fisher Scientific, Inc.), according to the manufacturer's protocol and RNA quality and concentration was detected by ultraviolet spectrophotometry (NanoDrop $^{\text {TM }}$ 2000; Thermo Fisher Scientific, Inc.). To detect the mRNA expression level of CASP2, total RNA $(1 \mu \mathrm{g})$ was reverse transcribed into cDNA using the TIANScript II cDNA First Strand Synthesis kit (Tiangen Biotech Co., Ltd., Beijing, China). qPCR was subsequently performed using the SuperReal PreMix (SYBR Green) kit (Tiangen Biotech Co., Ltd.) with the following primer pairs: CASP2 forward, 5'-GCAAACCTC AGGGAAACATTC-3' and reverse, 5'-TGTCGGCATACTGTT TCAGCA-3'; and GAPDH forward, 5'-AGGAGCGAGACC CCACTAACAT-3' and reverse, 5'-GTGATGGCATGGACT GTGGT-3'. The following thermocycling conditions were used for the qPCR: Initial denaturation at $95^{\circ} \mathrm{C}$ for $5 \mathrm{~min} ; 46$ cycles of $95^{\circ} \mathrm{C}$ for $20 \mathrm{sec}, 55^{\circ} \mathrm{C}$ for $20 \mathrm{sec}$ and $72^{\circ} \mathrm{C}$ for $30 \mathrm{sec}$ which was performed using an iQ5 Real-Time PCR system (Bio-Rad Laboratories, Inc., Hercules, CA, USA). CASP2 mRNA levels were quantified using the $2^{-\triangle \Delta C q}$ method and normalized to the internal reference gene GAPDH (18). To detect the expression level of miR-182-5p, miRNA was reverse transcribed into cDNA using the miRcute miRNA cDNA First Strand Synthesis kit (Tiangen Biotech Co., Ltd.). qPCR was performed using the miRcute miRNA qPCR Detection kit (Tiangen Biotech Co., Ltd.) and the following primer pairs: miR-182-5p forward, 5'-ACACTCCAGCTGGGTTTGGCAATGGTAGAACT-3' and reverse, 5'-TGGTGTCGTGGAGTCG-3'; and U6 forward, 5'-CTCGCTTCGGCAGCACA-3' and reverse, 5'-AACGCT TCACGAATTTGCGT-3'. The following thermocycling conditions were used for qPCR: Initial denaturation at $95^{\circ} \mathrm{C}$ for $5 \mathrm{~min} ; 46 \mathrm{cycles}$ of $95^{\circ} \mathrm{C}$ for $10 \mathrm{sec}, 56^{\circ} \mathrm{C}$ for $25 \mathrm{sec}$ and $72^{\circ} \mathrm{C}$ for $30 \mathrm{sec}$ which was performed using an iQ5 Real-Time PCR system (Bio-Rad Laboratories, Inc.). miR-182-5p expression levels were quantified using the $2^{-\Delta \Delta C q}$ method and normalized using U6 as an internal reference $(18,19)$. Each experiment was performed in triplicate.

MTT assay. Following transfection, cells were seeded into 96-well plates at a density of $2 \times 10^{3}$ cells/well and cultured for 24, 48 and 72 h. Following incubation, $20 \mu \mathrm{l}$ MTT (5 g/l; cat. no. JRDC000003; JRDUN Biotechnology, Co., Ltd., Shanghai, China) was added to each well and further incubated for $4 \mathrm{~h}$ 
at $37^{\circ} \mathrm{C}$. Each condition was assessed in triplicate. Following incubation, the culture medium was aspirated and dimethyl sulfoxide (150 $\mu \mathrm{l} /$ well) was added to dissolve the purple formazan crystals. The absorbance of each well was measured at $490 \mathrm{~nm}$ using a microplate reader (Bio-Rad Laboratories, Inc.) and cell proliferation curves were plotted. Each experiment was performed in triplicate.

Flow cytometry. Following transfection, cells $\left(1 \times 10^{6}\right)$ from each group were washed twice with pre-cooled PBS twice and cell apoptosis was analyzed by flow cytometry using the FITC Annexin V Apoptosis Detection Kit I (BD Biosciences, Franklin Lakes, NJ, USA), according to the manufacturer's protocol. Annexin V-positive staining were early apoptotic cells, those with propidium iodide-positive staining were necrotic cells and those with double positive staining were late apoptotic cells. Each experiment was performed in triplicate. CFlow Plus software (v1.0.264.15; BD Biosciences) was used to analysis the results.

Bioinformatics analysis. Bioinformatics prediction is a powerful tool for the study of miRNAs. Bioinformatics analysis was performed using miRanda (www.microrna. org/microrna/home.do), TargetScan (www.targetscan.org), PiTa (genie.weizmann.ac.il/pubs/mir07/mir07_data.html), RNAhybrid (bibiserv.techfak.uni-bielefeld.de/rnahybrid) and PICTA (pictar.mdc-berlin.de) to predict potential target genes of miR-182-5p.

Western blot analysis. Prior to lysis, tissue samples were ground into powder in liquid nitrogen, and cells were trypsinized and collected. Subsequently, total protein was extracted from tissue samples or cells using pre-cooled radioimmunoprecipitation assay lysis buffer $(600 \mu \mathrm{l} ; 50 \mathrm{mM}$ Tris-base, $1 \mathrm{mM}$ EDTA, $150 \mathrm{mM} \mathrm{NaCl}, 0.1 \%$ sodium dodecyl sulfate, $1 \%$ TritonX-100, 1\% sodium deoxycholate; Beyotime Institute of Biotechnology, Haimen, China) for $30 \mathrm{~min}$ on ice. The lysates were centrifuged at $10,000 \times \mathrm{g}$ for $10 \mathrm{~min}$ at $4^{\circ} \mathrm{C}$ and the supernatant was used to quantify protein concentration using a bicinchoninic acid protein concentration determination kit (cat. no. RTP7102; Real-Times Biotechnology Co., Ltd., Beijing, China). The samples were mixed with 5X SDS loading buffer prior to denaturation in a water bath at $100^{\circ} \mathrm{C}$ for $5 \mathrm{~min}$. Following denaturation, $20 \mu \mathrm{g}$ protein/lane was separated at $100 \mathrm{~V}$ via SDS-PAGE on a $10 \%$ gel. The separated proteins were transferred onto polyvinylidene difluoride membranes $(100 \mathrm{~V}, 2 \mathrm{~h})$ and blocked with $5 \%$ skimmed milk at room temperature for $1 \mathrm{~h}$. The membranes were incubated with primary antibodies: Rabbit anti-human CASP2 (1:1,000; cat. no. ab182657) or $\beta$-actin (1:5,000; cat. no. ab129348; both Abcam, Cambridge, UK) overnight at $4^{\circ} \mathrm{C}$. The membranes were washed three times for 15 min with PBS containing Tween ${ }^{\circledR}-20$. The membranes were incubated with goat anti-rabbit horseradish peroxidase (HRP)-conjugated secondary antibody (1:3,000; cat. no. ab6721; Abcam) for $1 \mathrm{~h}$ at room temperature. The membranes were washed three times for 15 min with PBS containing Tween ${ }^{\circledR}-20$. Protein bands were developed using the ECL Western Blotting Substrate kit (cat. no. ab65623; Abcam). Protein expression was quantified using Image Lab v3.0 software (Bio-Rad Laboratories, Inc.) with $\beta$-actin as the loading control. Each experiment was performed in triplicate.

ELISA. Peripheral blood was centrifuged at $1,000 \times \mathrm{g}$ for 10 min at $4^{\circ} \mathrm{C}$. The CASP2 Human ELISA kit (cat. no. KA2636; Abnova, Taipei, Taiwan) was used for the quantitative detection and measurement of CASP2 in serum. Briefly, standards (50 $\mu \mathrm{l})$ and samples (10 $\mu \mathrm{l}$ serum and $40 \mu \mathrm{l}$ diluent) were added to predefined wells and blank wells were left empty. In each well, for HRP-labeled conjugates $(100 \mu \mathrm{l})$ were added to each well and the plates were sealed and incubated at $37^{\circ} \mathrm{C}$ for $1 \mathrm{~h}$. Plates were washed 5 times and substrates A $(50 \mu \mathrm{l})$ and $\mathrm{B}(50 \mu \mathrm{l})$ were added to each well and incubated at $37^{\circ} \mathrm{C}$ for $15 \mathrm{~min}$. Following incubation, stop solution (50 $\mu \mathrm{l})$ was added to each well and the absorbance was measured within $15 \mathrm{~min}$ at $450 \mathrm{~nm}$ using a Multiskan FC (Thermo Fisher Scientific, Inc.). Each experiment was performed in triplicate.

Dual-luciferase reporter assay. The wild-type (WT) and mutant 3'-untranslated region (UTR) of CASP2 containing the seed regions of miR-182-5p were chemically synthesized in vitro and cloned into the pMIR-REPORT luciferase reporter plasmid (Promega Corporation, Madison, WI, USA) between the Spe-1 and Hind III restriction sites. 293T cells (Cell Bank, Chinese Academy of Sciences, Shanghai, China) were subsequently co-transfected with agomiR-182-5p (100 nM; forward, 5'-UUUGGCAAUGGUAGA ACUCACACU-3'; reverse, 3'-AAACCGUUACCAUCAAGAGUGUGA-5'; Sangon Biotech Co., Ltd.) and the WT or mutant 3'-UTR CASP2 luciferase reporter plasmids $(0.8 \mu \mathrm{g}) .293 \mathrm{~T}$ cells were transfected with agomiR-negative control (NC; forward, 5'-UUCUCCGAA CGUGUCACGUTT-3'; reverse, 3'-TTAAGAGGCUUGCAC AGUGCA-5'; Sangon Biotech Co., Ltd.) as a control. Following 24-h transfection, cells were lysed and luciferase activities were measured using the Dual-Luciferase Reporter Assay system (Promega Corporation) according to the manufacturer's protocol and luciferase activity was detected using a GloMax 20/20 luminometer (Promega Corporation). Firefly luciferase activity was normalized to Renilla luciferase activity and each experiment was performed in triplicate.

Statistical analysis. Data are presented as the mean \pm standard deviation. All statistical analyses were performed using SPSS statistical software (version 20.0; IBM Corp., Armonk, NY, USA). Data were tested for normality and statistical analysis among multiple groups was analyzed by one-way analysis of variance followed by Student-Newman-Keuls test. Comparison between NSCLC and adjacent normal tissue samples from patients with NSCLC was performed using a paired Student's t-test, while comparison between the experimental and control groups was carried out using an unpaired Student's t-test. $\mathrm{P}<0.05$ was considered to indicate a statistically significant difference.

\section{Results}

miR-182-5p expression is upregulated in NSCLC. To examine the expression pattern of miR-182-5p in NSCLC, the miR-182-5p expression level was determined by RT-qPCR. The miR-182-5p expression level was significantly increased in NSCLC tissue samples compared with adjacent normal tissue samples 
A

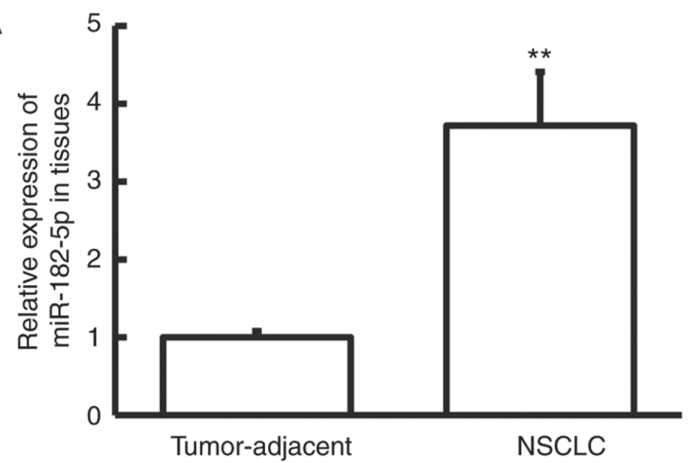

$\mathrm{B}$

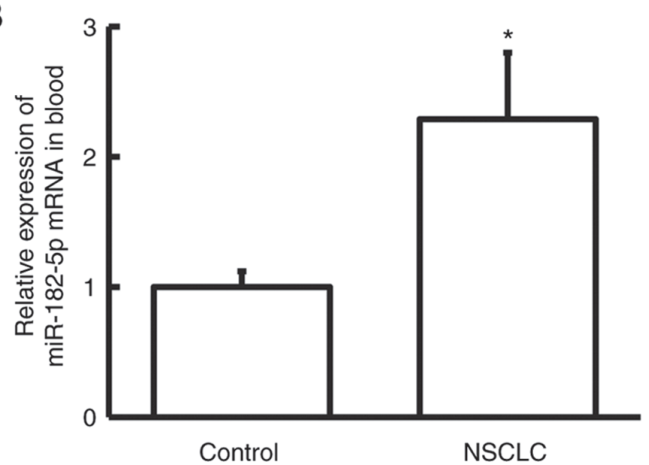

Figure 1. miR-182-5p expression in NSCLC tissue samples and peripheral blood. (A) The relative expression level of miR-182-5p in NSCLC and adjacent normal tissue samples from patients with NSCLC. ${ }^{* *} \mathrm{P}<0.01$ vs. tumor-adjacent. (B) The relative expression level of miR-182-5p in peripheral blood samples obtained from patients with NSCLC and healthy controls. "P<0.05 vs. control. miR, microRNA; NSCLC, non-small cell lung cancer.

A
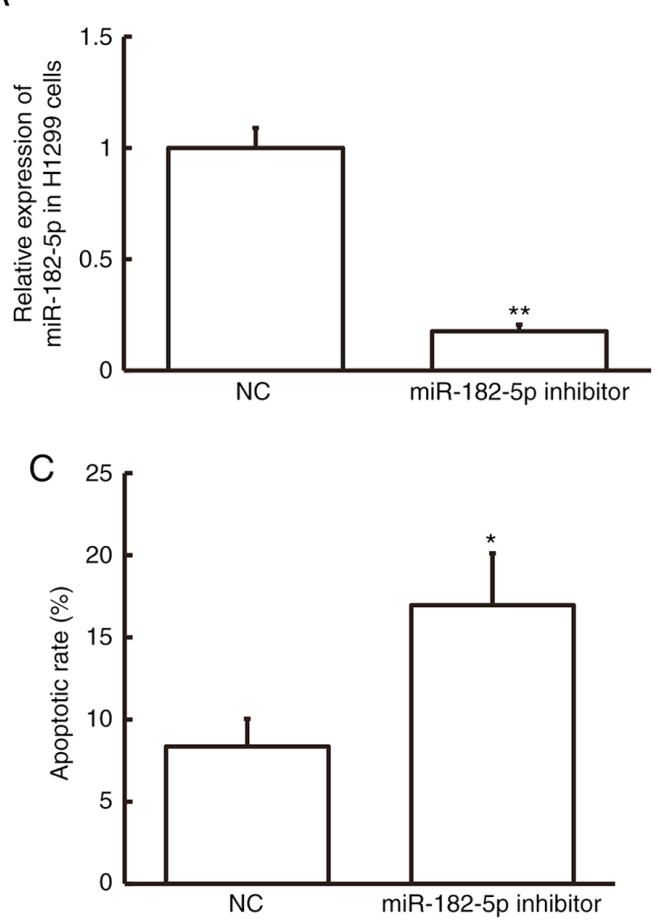

B

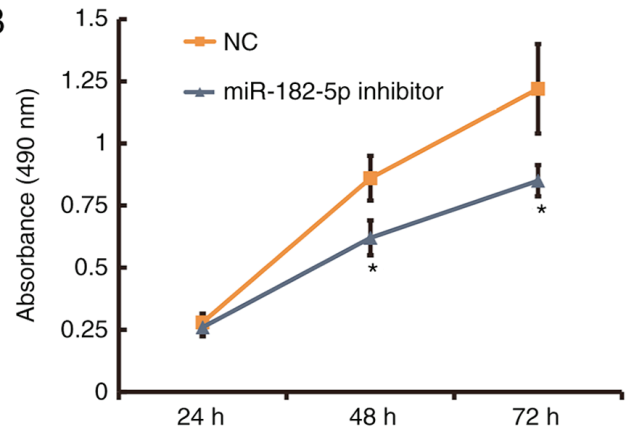

Figure 2. Effect of miR-182-5p on NSCLC cell proliferation and apoptosis. (A) The relative expression level of miR-182-5p was determined by reverse transcription-quantitative polymerase chain reaction in the NSCLC cell line H1299 following transfection with miR-182-5p inhibitor or NC. (B) MTT assay was used to examine cell proliferation of H1299 cells transfected with NC or miR-182-5p inhibitor for 24, 48 and 72 h. (C) Flow cytometry was used to detect apoptosis in $\mathrm{H} 1299$ cells transfected with NC or miR-182-5p inhibitor. ${ }^{*} \mathrm{P}<0.05$ and ${ }^{* *} \mathrm{P}<0.01$ vs. NC. miR, microRNA; NSCLC, non-small cell lung cancer; NC, negative control.

(P<0.01; Fig. 1A). Similarly, the miR-182-5p expression level was significantly increased in peripheral blood samples from patients with NSCLC compared with healthy controls $(\mathrm{P}<0.05$; Fig. 1B). These results suggest that miR-182-5p expression is upregulated in NSCLC, and therefore miR-182-5p may exert its biological function in NSCLC.

Inhibition of miR-182-5p expression suppresses cell proliferation and promotes cell apoptosis in NSCLC cells. To examine the effect of miR-182-5p on NSCLC cell proliferation, the MTT assay was performed in the NSCLC cell line H1299 following transfection with miR-182-5p inhibitor. The miR-182-5p level was significantly decreased in H1299 cells following transfection with miR-182-5p inhibitor compared with the $\mathrm{NC}(\mathrm{P}<0.01$; Fig. 2A). MTT assay demonstrated that cell proliferation was significantly decreased in H1299 cells following 48 and 72-h transfection with miR-182-5p inhibitor compared with the NC $(\mathrm{P}<0.05$; Fig. 2B). Flow cytometry demonstrated that the rate of apoptosis was significantly increased in H1299 cells following transfection with miR-182-5p inhibitor compared with the $\mathrm{NC}(\mathrm{P}<0.05$; Fig. $2 \mathrm{C})$. These results suggest that inhibition of miR-182-5p expression suppresses cell proliferation and promotes cell apoptosis in NSCLC cells and therefore miR-182-5p may serve a role in NSCLC progression. 
$5^{\prime}$

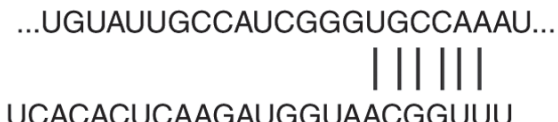

$3^{\prime}$

UCACACUCAAGAUGGUAACGGUUU

$\operatorname{miR}-182-5 p$

Figure 3. CASP2 is a direct target gene of miR-182-5p. Bioinformatics was used to predict the miR-182-5p binding site in the wild-type 3'-untranslated region of CASP2. CASP2, caspase 2; miR, microRNA.

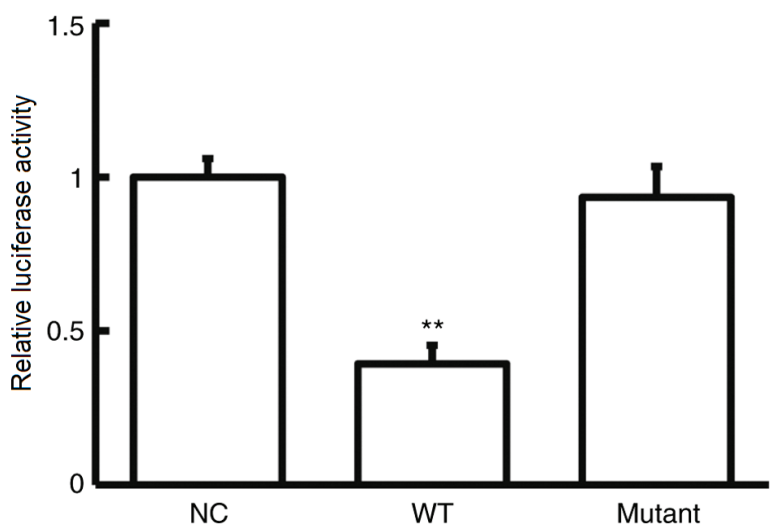

Figure 4. Direct interaction between miR-182-5p and CASP2. Luciferase activity of WT or mutant CASP2 3'-UTR. The dual-luciferase reporter assay demonstrated that miR-182-5p inhibited the activity of wild-type CASP2 3'-UTR in 293 T cells. * ${ }^{*}<0.05$ vs. NC. miR, microRNA; CASP2, caspase 2; $\mathrm{NC}$, negative control; WT, wild-type; UTR, untranslated region.

CASP 2 is a direct target gene of miR-182-5p in NSCLC cells. To further investigate the role of miR-182-5p in NSCLC, potential targets of miR-182-5p were examined. Bioinformatics analysis showed that CASP2 was identified as a potential target gene of miR-182-5p (Fig. 3). The dual-luciferase reporter assay was used verify the interaction between miR-182-5p and the 3'-UTR of CASP2. The current study demonstrated that agomiR-182-5p overexpression significantly decreased pMIR-REPORT-WT luciferase activity compared with the $\mathrm{NC}(\mathrm{P}<0.05$; Fig. 4). By contrast, co-transfection with agomiR-182-5p and the pMIR-REPORT-mutant luciferase reporter plasmid had no significant effect on luciferase activity. These results indicate that miR-182-5p can directly bind with the 3'-UTR of CASP2 .

CASP2 expression is downregulated in NSCLC tissue and peripheral blood samples from patients with NSCLC. To investigate CASP2 expression in NSCLC, the mRNA and protein expression levels of CASP2 were determined by RT-qPCR, and western blotting and ELISA, respectively. The mRNA and protein expression levels of CASP2 were significantly decreased in NSCLC tissue samples compared with adjacent normal tissue samples from patients with NSCLC $(\mathrm{P}<0.05$; Fig. 5A and B). Similarly, RT-qPCR and ELISA demonstrated that the mRNA and protein expression levels of CASP2 were significantly decreased in peripheral blood samples from patients with NSCLC compared with healthy controls $(\mathrm{P}<0.01$ and $\mathrm{P}<0.05$, respectively; Fig. $5 \mathrm{C}$ and $\mathrm{D})$. These results suggest that CASP2 expression is downregulated in patients with NSCLC.

miR-182-5p regulates NSCLC cell proliferation and apoptosis through regulation of CASP 2 expression. To investigate the
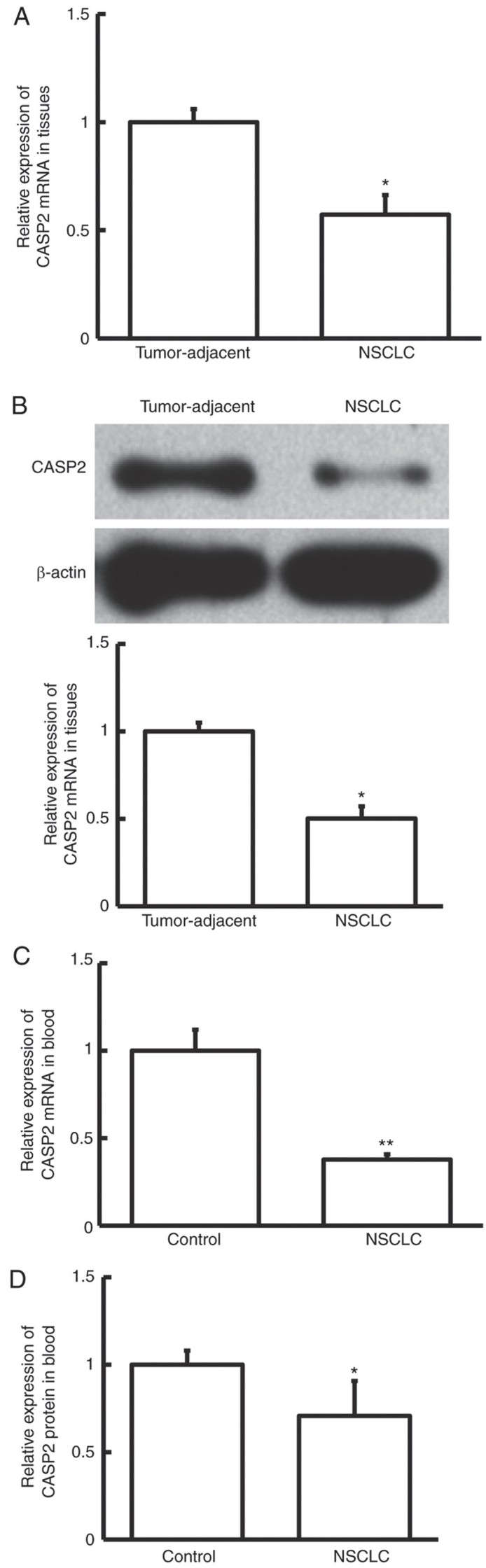

Figure 5. CASP2 expression in NSCLC tissue samples and peripheral blood. The relative (A) mRNA and (B) protein expression level of CASP2 in NSCLC and adjacent normal tissue samples from patients with NSCLC. ${ }^{\text {"P }}<0.05$ vs. tumor-adjacent. The relative (C) mRNA and (D) protein expression of CASP2 in peripheral blood samples obtained from patients with NSCLC and healthy controls. ${ }^{~} \mathrm{P}<0.05$ and ${ }^{* *} \mathrm{P}<0.01$ vs. control. CASP2, caspase 2 ; NCSLC, non-small cell lung cancer. 


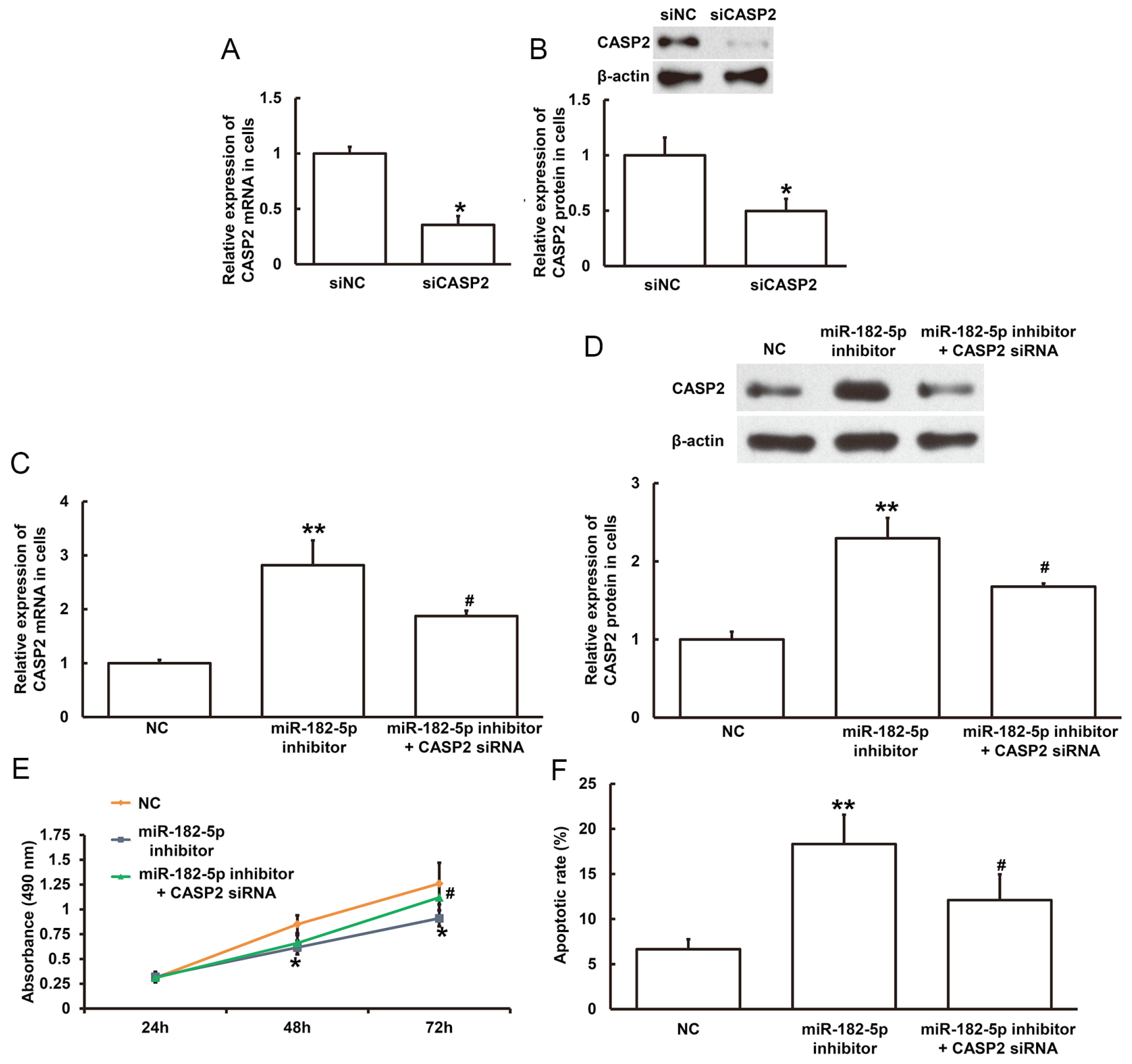

Figure 6. Effect of miR-182-5p on CASP2 expression and NSCLC cell proliferation. The relative (A) mRNA and (B) protein expression level of CASP2 in H1299 cells following transfection with siNC or CASP2 siRNA. " $\mathrm{P}<0.05$ compared with siNC group. The relative (C) mRNA and (D) protein expression level of CASP2 in H1299 cells following transfection with NC, miR-182-5p inhibitor or miR-182-5p inhibitor and CASP2 siRNA. ${ }^{* *} \mathrm{P}<0.01$ vs. NC; ${ }^{\prime} \mathrm{P}<0.05$ vs. miR-182-5p inhibitor. (E) MTT assay was used to examine cell proliferation of H1299 cells transfected with NC, miR-182-5p inhibitor or miR-182-5p inhibitor and CASP2 siRNA for 24, 48 and 72 h. " $\mathrm{P}<0.05$ vs. NC; ${ }^{~} \mathrm{P}<0.05$ vs. miR-182-5p inhibitor. (F) Flow cytometry was used to detect apoptosis in H1299 cells transfected with NC, miR-182-5p inhibitor or miR-182-5p inhibitor and CASP2 siRNA. ${ }^{* *} \mathrm{P}<0.01$ vs. NC; ${ }^{*} \mathrm{P}<0.05$ vs. miR-182-5p inhibitor. miR, microRNA; CASP2, caspase 2; NSCLC, non-small cell lung cancer; siNC, negative control siRNA; siRNA, small interfering RNA; NC, negative control.

underlying mechanism of miR-182-5p on NSCLC cell proliferation, the MTT assay was performed in the NSCLC cell line H1299 following transfection with miR-182-5p inhibitor with or without CASP2. Initially, transfection efficiency was examined in H1299 cells. The mRNA and protein expression levels of CASP2 were significantly decreased following transfection with CASP2 siRNA compared with siNC $(\mathrm{P}<0.05$; Fig. 6A and B). In addition, inhibition of miR-182-5p significantly increased CASP2 mRNA and protein expression levels compared with NC $(\mathrm{P}<0.01$; Fig. $6 \mathrm{C}$ and $\mathrm{D})$. Furthermore, co-transfection with miR-182-5p inhibitor and CASP2 siRNA significantly decreased CASP2 mRNA and protein expression levels compared with miR-182-5p inhibitor alone $(\mathrm{P}<0.05$;
Fig. 6C and D). MTT assay demonstrated that cell proliferation was significantly decreased in H1299 cells following 48-and 72-h transfection with miR-182-5p inhibitor compared with the $\mathrm{NC}(\mathrm{P}<0.05$; Fig. $6 \mathrm{E})$; however, cell proliferation was significantly increased following 72-h co-transfection with CASP2 siRNA compared with miR-182-5p inhibitor alone $(\mathrm{P}<0.05$; Fig. 6E). Flow cytometry demonstrated that the rate of apoptosis was significantly increased in H1299 cells following transfection with miR-182-5p inhibitor compared with the $\mathrm{NC}(\mathrm{P}<0.01$; Fig. 6F), however the rate of apoptosis was significantly decreased following co-transfection with CASP2 siRNA compared with miR-182-5p inhibitor alone $(\mathrm{P}<0.05$; Fig. 6F). Taken together, these results suggest that miR-182-5p 
may regulate $\mathrm{H} 1299$ cell proliferation and apoptosis by directly binding with the 3'-UTR of CASP2 to regulate CASP2 expression.

\section{Discussion}

Lung cancer can be divided into two histologic classes: small-cell lung cancer (SCLC) and NSCLC (20,21). NSCLC accounts for $\sim 80-85 \%$ of all lung cancer cases, with a 5-year survival rate of $16 \%$ (22). In the early (occult) stage, lung cancer is difficult to diagnose and most patients with lung cancer are diagnosed with late stage lung cancer (23). Although surgery and/or chemoradiotherapy can prolong the survival time of patients with lung cancer, prognosis remains poor (24). In recent years, the development of molecular targeted drugs has improved the survival rates of patients with advanced lung cancer, however due to the relatively small subset of NSCLC patients and high drug resistance, the application of molecular targeted drugs has had limited success (25-28). It is therefore important to investigate the molecular mechanisms underlying the occurrence and development of lung cancer, and identify novel diagnostic and therapeutic targets with high specificity.

Several studies have demonstrated the important roles of miRNAs in the initiation and progression of $\operatorname{NSCLC}(22,29,30)$. In addition, previous studies revealed that miR-182-5p may serve different roles in different types of cancer, including renal cell carcinoma, neuroblastoma, liver cancer, ovarian cancer, prostate cancer and bladder cancer (11,12,31-37). The expression of miR-182-5p is associated with cell proliferation and prognosis in renal cell carcinoma $(11,32)$, as well as cell differentiation and apoptosis in neuroblastoma (31). In prostate cancer, the expression of miR-182-5p is associated with cancer cell proliferation and invasion (33). In addition, the expression of miR-182-5p is known to have a carcinogenic effect in bladder cancer (36). In the present study, miR-182-5p expression was upregulated in tumor tissue and peripheral blood samples from patients with NSCLC and the MTT assay demonstrated that inhibition of miR-182-5p decreased NSCLC cell proliferation. These results suggest that miR-182-5p may be involved in NSCLC progression.

Bioinformatics analysis was used to identify CASP2 as a potential target gene of miR-182-5p. CASP2 serves important roles in stress-induced apoptosis, and CASP2 activation occurs in response to apoptosis-stimulating factors, which include tumor necrosis factor- $\alpha(38,39)$, Fas $(40)$ and growth factor deficiency $(41,42)$. In the current study, CASP2 expression is downregulated in tumor tissue and peripheral blood samples from patients with NSCLC, which suggests that CASP2-mediated apoptosis may be involved in the pathogenesis of NSCLC. In addition, the current study demonstrated that miR-182-5p may regulate NSCLC cell proliferation and apoptosis via the regulation of CASP2 expression, as decreased cell proliferation induced by the inhibition of miR-182-5p was partially restored by CASP 2 siRNA, possibly due to transfection efficiency and cell type (18). The dual-luciferase reporter assay demonstrated that miR-182-5p interacts directly with the 3'-UTR of CASP2 mRNA to regulate CASP2 expression.

In the current study, both tumor tissue and peripheral blood samples were examined, and the results demonstrated the potential diagnostic application of blood sample collection when screening patients for NSCLC $(31,32)$. The present study demonstrated that upregulated miR-182-5p expression in tumor tissue and peripheral blood samples from patients with NSCLC may be associated with the downregulation of CASP2 expression. In addition, miR-182-5p may regulate NSCLC proliferation via CASP2, however future studies are required to further clarify the association between miR-182-5p and CASP2 in NSCLC.

\section{Acknowledgements}

Not applicable.

\section{Funding}

The present study was supported by a grant from the Science and Technology Project of Shandong Education Department (grant no. J17KB088).

\section{Availability of data and materials}

The datasets used and/or analyzed during the present study are available from the corresponding author on reasonable request.

\section{Authors' contributions}

LY, YD and MX designed the study. LY, YD, ZS, HC, XL, QW PG and YQ performed the experiments. LY, YD, ZS and $\mathrm{HC}$ analyzed the data. All authors collaborated to interpret the results and prepare the manuscript. All authors read and approved the final manuscript.

\section{Ethical approval and consent to participate}

The present study was approved by the Ethics Committee of Qilu Medical University (Zibo, China). Written informed consent was obtained from all patients or their families.

\section{Patient consent for publication}

Written informed consents for publication of any associated data and accompanying images were obtained from all patients or their parents, guardians or next of kin.

\section{Competing interests}

The authors declare that they have no competing interests.

\section{References}

1. Torre LA, Bray F, Siegel RL, Ferlay J, Lortet-Tieulent J and Jemal A: Global cancer statistics, 2012. CA Cancer J Clin 65: 87-108, 2015.

2. Kang CG, Lee HJ, Kim SH and Lee EO: Zerumbone suppresses osteopontin-induced cell invasion through inhibiting the FAK/AKT/ROCK pathway in human non-small cell lung cancer A549 Cells. J Nat Prod 79: 156-160, 2016.

3. Xu YJ, Du Y and Fan Y: Long noncoding RNAs in lung cancer: What we know in 2015. Clin Transl Oncol 18: 660-665, 2016.

4. She J, Yang P, Hong Q and Bai C: Lung cancer in China: Challenges and interventions. Chest 143: 1117-1126, 2013.

5. Liu Q and Zhou Q: The challenges of lung cancer in China. J Cancer Res Ther 9 (Suppl 2): S65-S66, 2013. 
6. Nakanishi K, Mizuno T, Sakakura N, Kuroda H, Shimizu J Hida T, Yatabe Y and Sakao Y: Salvage surgery for small cell lung cancer after chemoradiotherapy. Jpn J Clin Oncol 1: 389-392, 2019.

7. Gamazon ER, Trendowski MR, Wen Y, Wing C, Delaney SM, Huh W, Wong S, Cox NJ and Dolan ME: Gene and MicroRNA perturbations of cellular response to pemetrexed implicate biological networks and enable imputation of response in lung adenocarcinoma. Sci Rep 8: 733, 2018.

8. Inoue K: MicroRNA function in animal development. Tanpakushitsu Kakusan Koso 52: 197-204, 2007 (In Japanese).

9. Liu G, Li YI and Gao X: Overexpression of microRNA-133b sensitizes non-small cell lung cancer cells to irradiation through the inhibition of glycolysis. Oncol Lett 11: 2903-2908, 2016.

10. Jiang LP, Zhu ZT and He CY: Expression of miRNA-26b in the diagnosis and prognosis of patients with non-small-cell lung cancer. Future Oncol 12: 1105-1115, 2016.

11. Fan Y, Li H, Ma X, Go Y, Bao X, Du Q, Ma M, Liu K, Yao Y, Huang Q, et al: Dicer suppresses the malignant phenotype in VHL-deficient clear cell renal cell carcinoma by inhibiting HIF-2 $\alpha$. Oncotarget 7: 18280-18294, 2016.

12. Assal RA, El Tayebi HM, Hosny KA, Esmat G and Abdelaziz AI: A pleiotropic effect of the single clustered hepatic metastamiRs miR-96-5p and miR-182-5p on insulin-like growth factor II insulin-like growth factor-1 receptor and insulin-like growth factor-binding protein-3 in hepatocellular carcinoma. Mol Med Rep 12: 645-650, 2015.

13. Dorstyn L, Puccini J, Wilson CH, Shalini S, Nicola M, Moore S and Kumar S: Caspase-2 deficiency promotes aberrant DNA-damage response and genetic instability. Cell Death Differ 19: 1288-1298, 2012.

14. Wang Y, Liu C, Wang J, Zhang Y and Chen L: Iodine-131 induces apoptosis in human cardiac muscle cells through the p53/Bax/caspase-3 and PIDD/caspase-2/tBID/cytochrome c/caspase-3 signaling pathway. Oncol Rep 38: 1579-1586, 2017.

15. Jang TH, Lee SJ, Woo CH, Lee KJ, Jeon JH, Lee DS, Choi K, Kim IG, Kim YW, Lee TJ and Park HH: Inhibition of genotoxic stress induced apoptosis by novel TAT-fused peptides targeting PIDDosome. Biochem Pharmacol 83: 218-227, 2012.

16. Liu J, Dou Y and Sheng M: Inhibition of microRNA-383 has tumor suppressive effect in human epithelial ovarian cancer through the action on caspase-2 gene. Biomed Pharmacother 83: 1286-1294, 2016

17. Makwana K, Patel SA, Velingkaar N, Ebron JS, Shukla GC and Kondratov RVKV: Aging and calorie restriction regulate the expression of miR-125a-5p and its target genes Stat3, Casp2 and Stard13. Aging (Albany NY) 9: 1825-1843, 2017.

18. Xia D, Li X, Niu Q, Liu X, Xu W, Ma C, Gu H, Liu Z, Shi L, Tian X, et al: MicroRNA-185 suppresses pancreatic cell proliferation by targeting transcriptional coactivator with PDZ-binding motif in pancreatic cancer. Exp Ther Med 15: 657-666, 2018.

19. He X, Ping J and Wen D: MicroRNA-186 regulates the invasion and metastasis of bladder cancer via vascular endothelial growth factor C. Exp Ther Med 14: 3253-3258, 2017.

20. Arocho A, Chen B, Ladanyi M and Pan Q: Validation of the 2-DeltaDeltaCt calculation as an alternate method of data analysis for quantitative PCR of BCR-ABL P210 transcripts. Diagn Mol Pathol 15: 56-61, 2006.

21. Oser MG, Niederst MJ, Sequist LV and Engelman JA Transformation from non-small-cell lung cancer to small-cell lung cancer: Molecular drivers and cells of origin. Lancet Oncol 16: e165-e172, 2015.

22. Chen G, Umelo IA, Lv S, Teugels E, Fostier K, Kronenberger P, Dewaele A, Sadones J, Geers C and De Greve J: miR-146a inhibits cell growth, cell migration and induces apoptosis in non-small cell lung cancer cells. PLoS One 8: e60317, 2013

23. Hofman P: The challenges of evaluating predictive biomarkers using small biopsy tissue samples and liquid biopsies from non-small cell lung cancer patients. J Thorac Dis 11 (Suppl 1): S57-S64, 2019.

24. Bray F, Ferlay J, Soerjomataram I, Siegel RL, Torre LA and Jemal A: Global cancer statistics 2018: GLOBOCAN estimates of incidence and mortality worldwide for 36 cancers in 185 countries. CA Cancer J Clin 68: 394-424, 2018.

25. Castanon E, Martin P, Rolfo C, Fusco JP, Ceniceros L, Legaspi J, Santisteban M and Gil-Bazo I: Epidermal growth factor receptor targeting in non-small cell lung cancer: Revisiting different strategies against the same target. Curr Drug Targets 15: 1273-1283, 2014.
26. Chen G, Kronenberger P, Teugels E, Umelo IA and De Greve J: Effect of siRNAs targeting the EGFR T790M mutation in a non-small cell lung cancer cell line resistant to EGFR tyrosine kinase inhibitors and combination with various agents. Biochem Biophys Res Commun 431: 623-629, 2013.

27. Chen G, Kronenberger P, Teugels E, Umelo IA and De Greve J: Targeting the epidermal growth factor receptor in non-small cell lung cancer cells: The effect of combining RNA interference with tyrosine kinase inhibitors or cetuximab. BMC Med 10: 28, 2012.

28. Chen G, Noor A, Kronenberger P, Teugels E, Umelo IA and De Greve J: Synergistic effect of afatinib with su11274 in non-small cell lung cancer cells resistant to gefitinib or erlotinib. PLoS One 8: e59708, 2013.

29. Lan D, Zhang X, He R, Tang R, Li P, He Q and Chen G: MiR-133a is downregulated in non-small cell lung cancer: A study of clinical significance. Eur J Med Res 20: 50, 2015.

30. Zhang X, Li P, Rong M, He R, Hou X, Xie Y and Chen G: MicroRNA-141 is a biomarker for progression of squamous cell carcinoma and adenocarcinoma of the lung: Clinical analysis of 125 patients. Tohoku J Exp Med 235: 161-169, 2015.

31. Rihani A, Van Goethem A, Ongenaert M, De Brouwer S, Volders PJ, Agarwal S, De Preter K, Mestdagh P, Shohet J, Speleman F, et al: Genome wide expression profiling of p53 regulated miRNAs in neuroblastoma. Sci Rep 5: 9027, 2015.

32. Chen L, Ma H, Hu H, Gao L, Wang X, Ma J, Gao Q, Liu B, Zhou G and Liang C: Special role of Foxp3 for the specifically altered microRNAs in Regulatory T cells of HCC patients. BMC Cancer 14: 489, 2014.

33. Xu X, Wu J, Li S, Hu Z, Xu X, Zhu Y, Liang Z, Wang X, Lin Y, Mao Y, et al: Downregulation of microRNA-182-5p contributes to renal cell carcinoma proliferation via activating the $\mathrm{AKT} / \mathrm{FOXO} 3 \mathrm{a}$ signaling pathway. Mol Cancer 13: 109, 2014.

34. Wang L, Zhu MJ, Ren AM, Wu HF, Han WM, Tan RY and Tu RQ: A ten-microRNA signature identified from a genome-wide microRNA expression profiling in human epithelial ovarian cancer. PLoS One 9: e96472, 2014.

35. Hirata H, Ueno K, Shahryari V, Deng G, Tanaka Y, Tabatabai ZL, Hinoda Y and Dahiya R: MicroRNA-182-5p promotes cell invasion and proliferation by down regulating FOXF2, RECK and MTSS1 genes in human prostate cancer. PLoS One 8: e55502, 2013.

36. Hirata H, Ueno K, Shahryari V, Tanaka Y, Tabatabai ZL, Hinoda Y and Dahiya R: Oncogenic miRNA-182-5p targets Smad4 and RECK in human bladder cancer. PLoS One 7: e51056, 2012.

37. Tsuchiyama $K$, Ito $H$, Taga $M$, Naganuma S, Oshinoya $Y$, Nagano K, Yokoyama $\mathrm{O}$ and Itoh $\mathrm{H}$ : Expression of microRNAs associated with Gleason grading system in prostate cancer: miR-182-5p is a useful marker for high grade prostate cancer. Prostate 73: 827-834, 2013.

38. Suzuki Y and Farbman AI: Tumor necrosis factor-alpha-induced apoptosis in olfactory epithelium in vitro: Possible roles of caspase 1 (ICE), caspase 2 (ICH-1), and caspase 3 (CPP32). Exp Neurol 165: 35-45, 2000.

39. Lv XX, Yu XH, Wang HD, Yan YX, Wang YP, Lu DX, Qi RB, $\mathrm{Hu}$ CF and Li HM: Berberine inhibits norepinephrine-induced apoptosis in neonatal rat cardiomyocytes via inhibiting ROS-TNF- $\alpha$-caspase signaling pathway. Chin J Integr Med 19: 424-431, 2013.

40. Droin N, Bichat F, Rebe C, Wotawa A, Sordet O, Hammann A, Bertrand R and Solary E: Involvement of caspase-2 long isoform in Fas-mediated cell death of human leukemic cells. Blood 97: 1835-1844, 2001

41. Jean YY, Ribe EM, Pero ME, Moskalenko M, Iqbal Z, Marks LJ, Greene LA and Troy CM: Caspase-2 is essential for c-Jun transcriptional activation and Bim induction in neuron death. Biochem J 455: 15-25, 2013

42. Troy CM, Rabacchi SA, Hohl JB, Angelastro JM, Greene LA and Shelanski ML: Death in the balance: Alternative participation of the caspase- 2 and -9 pathways in neuronal death induced by nerve growth factor deprivation. J Neurosci 21: 5007-5016, 2001.

This work is licensed under a Creative Commons Attribution-NonCommercial-NoDerivatives 4.0 International (CC BY-NC-ND 4.0) License. 